\title{
A Pragmatic Study on the Functions of Vague Language in Commercial Advertising
}

\author{
Zhu Wenzhong ${ }^{1} \&$ Li Jingyi ${ }^{2}$ \\ ${ }^{1}$ Professor, School of English for International Business, Guangdong University of Foreign Studies, China \\ ${ }^{2}$ Graduate Student, School of English for International Business, Guangdong University of Foreign Studies, \\ China \\ Correspondence: Li Jingyi, School of English for International Business, Guangdong University of Foreign \\ Studies, Baiyun Avenue, Guangzhou 510420, China. E-mail: 549268391@qq.com
}

Received: March 16, 2013 Accepted: April 11, 2013 Online Published: May 8, 2013

doi:10.5539/elt.v6n6p103 URL: http://dx.doi.org/10.5539/elt.v6n6p103

This paper is sponsored by the 2012 Project of Postgraduate Teaching Reform (12GWYJSJG - 10) of Guangdong University of Foreign Studies, and the 2012 project of Higher Education Teaching Quality and Reform (222-GK120063) of Guangdong Provincial Department of Education.

\begin{abstract}
Vagueness is one of the basic attributes of natural language. This is the same to advertising language. Vague language is a subject of increasing interest, and both foreign and domestic studies have attained success in it. Nevertheless, the study on the application of vague language in the context of English commercial advertising is relatively sparse. Since the effectiveness of communication of commercial advertisements is one of the greatest concerns of advertisers, this paper is to demonstrate the functions of vague language in commercial advertising, which is a communicative factor in the effectiveness of advertisements, through analysis of vagueness in advertisements under the guidance of pragmatics, Grice's Cooperative Principle and Conversational Implicature in particular. The paper shows that vague language in commercial advertising plays both positive and negative roles. Its positive functions include improving the flexibility of communication, enhancing the persuasiveness of communication and ensuring the accuracy of information whereas its negative functions cover misleading readers and making them subject to false understanding.
\end{abstract}

Keywords: vague language, commercial advertising, Cooperative Principle, Conversational Implicature, pragmatic functions

\section{Introduction}

\subsection{Rationale and Significance of the Study}

In the information age, speed and efficiency are highly valued. Advertising, as a means to convey information to the public, is capable of spreading information through mass media to a large number of people within a very limited period. Therefore, advertising is omnipresent in today's society. Language, as one of the essential elements which include images, colours, sound, etc. to express an advertisement, is of great significance to the effectiveness of communication of the advertisements.

Vagueness is one of the basic properties of natural language (Cao \& Gao, 2007). So is it with advertising language. Vague language is a common linguistic phenomenon in advertising, and it is gaining increasing attention in academic research. Study of vague language in the context of commercial advertising is relatively sparse, and this paper will focus on vague language in English commercial advertising. Through introduction of relevant theories on vague language and explication of the application of vagueness in English commercial advertisements from the perspective of a pragmatic theory, i.e. Grice's Cooperative Principle and Conversational Implicature, the functions of vague language in commercial advertising and the copywriters' motives to apply vague language will be revealed.

As globalization is a current trend in the business field, English, a language enjoying international popularity, is widely used in commercial advertising by international corporations. The success of advertisements can be a crucial factor for the success of the corporations' business. Hence, the study on the functions of vague language 
in English commercial advertising is of referential value to the corporations as it sheds some light on the design of a successful advertisement in terms of vague language. Furthermore, the study may offer the readers certain knowledge about vague language to assist them in realizing why and how it is applied, so as to help them safeguard themselves against an advertiser's ill purpose in the case that the advertiser deliberately exploits vague language to mislead or even deceive the readers for the sake of commercial interests.

\subsection{Research Questions}

This paper is to focus on vague language and its application in the context of English commercial advertising. Thus, the following questions will be involved.

1. What is vague language?

2. Why is it applied in English commercial advertising?

3. What pragmatic functions does it cause in English commercial advertising?

\subsection{Research Methodology}

The present study is a qualitative one. The examples in this paper adopted for the purpose of analyzing vague language in English commercial advertising are mostly selected from the commercial advertisements in the newspapers in the English-speaking countries, USA, UK, Australia, Canada included. The other examples are taken from advertising literature. After the collection work is completed, the examples, especially the headlines and the slogans thereof which generally serve the function of catching the readers' eyes, are studied under the guidance of Grice's theory. In this way, the motives of copywriters to use vague language and the functions of the application of that can be detailedly explored.

\section{Literature Review}

The root of the word "advertise" is "advertere", a Latin word meaning to warn, or to call attention to. Advertising is "the non-personal communication of information, usually paid for and usually persuasive in nature, about products (goods and services) or ideas by identified sponsors through various media" (Bovee \& Arens, 1992). Advertising can be categorized into the commercial type and the noncommercial one. As for the commercial advertising, it "promotes goods, services, or ideas for a business with the expectation of making a profit". To achieve their commercial purposes, advertisers have to make great efforts in attracting the consumers, especially in a way that will trigger their desire for consumption.

As a tool of communication, language is one of the essentials in advertisements, to which the copywriters usually pay much attention so as to make the communication a persuasive and effective one. Vagueness is one of the attributes of both language and advertising language. "Advertising language is loaded language. Its primary aim is to attract our attention and dispose us favorably towards the product or service on offer" (Dyer, 1995). In advertisements, such language usages as descriptive modifiers, elliptical sentences, etc. are widely seen due to the nature of loaded language. These language usages are subject to indeterminacy, that is, vagueness.

Vagueness is a common linguistic phenomenon in advertising. Then what is vague language? Scholars have viewed and studied vague language in different ways.

Pierce is often regarded as the originator of vagueness in language. He holds that "a proposition is vague where there are possible states of things concerning which it is intrinsically uncertain whether, had they been contemplated by the speaker, he would have regarded them as excluded or allowed by the proposition..." (Cited in Channell, 2000). In other words, a speaker may produce utterances without deciding whether certain facts are excluded or allowed by him.

Vague language is defined as words or phrases "which deliberately refer to people and things in a non-specific, imprecise way" (Carter \& McCarthy, 2006, cited in Cutting, 2007). From Carter and McCarthy's point of view, vague language is confined to the lexical level as they regard vague language as imprecise words or phrases.

Channel affirms that an expression is vague if (a) it can be contrasted with another word or expression which appears to render the same proposition, (b) it is purposely and unabashedly vague or (c) the meaning arises from intrinsic uncertainty (Channel, 1994, cited in Cutting, 2007).

Trappes-Lomax argues that vague language must be purposive, but it need not be unabashedly vague since disguising a vague intent may be a self-protective device (Cutting, 2007). His understanding of vague language diverges from Channel's, which considers an expression purposely and unabashedly made vague to be one of the circumstances where vagueness occurs. In Trappes-Lomax's opinion, vague language refers to "any purposive choice of language designed to make the degree of accuracy, preciseness, certainty, or clarity with which a 
referent or situation is described less than it might have been".

Cook studies vague language in the poetry context. He sees vague language as ambiguity and uncertainty of truth, as indeterminacy and imprecision (Cited in Cutting, 2007). He considers indeterminacy as a literary virtue.

In addition, some study vague language from a semantic perspective while some consider it as a pragmatic phenomenon. Ye (2012) summarizes that there are three branches in the study of semantic vagueness: semantic vagueness that is a reflection of the vagueness in the world; semantic vagueness that is a reflection of the vagueness in people's minds and of the limitation of people's conceptualization; and semantic vagueness which is a reflection of the vagueness in the language system itself. Ye refutes the idea that meaning is vague and maintains instead that meaning is clear. In his opinion, vagueness is a result of the limitation of human's senses and such a kind of vagueness cannot justify the reasonableness of the theories of semantic vagueness. He (2000) studies vague language from a pragmatic view. He considers vagueness as the language property of indeterminacy. He thinks that the study of vagueness in language is significant only when vagueness is analyzed from the perspectives of language use and comprehension. "The meaning of a single word which is indeterminate can be determined only in the field of pragmatics, that is to say, when that word is placed in a specific context".

From above, we can find that there are numerous statements concerning the definition of vague language, and that the ultimate definition has not been agreed on yet. In this paper, He's understanding of vague language as the language property of indeterminacy is to be adopted as the definition of vague language.

As regards the causes of vague language, Ullmann (1962) attributes vagueness to four factors: (a) generic character of words; (b) meaning is never homogeneous (it is context-bound); (c) lack of clear-cut boundaries in the non-linguistic world; and (d) lack of familiarity with what the words stand for (Cited in Channell, 2000).

Crystal and Davy (1975) give four reasons for vagueness: (a) the speaker forgets the right word on account of memory loss; (b) the language has no suitable word, or the speaker does not know it; (c) the subject of the conversation is not such that it requires precision, and an approximation or characterization will do; and (d) the choice of a vague item is deliberate to maintain the atmosphere (Cited in Qiao, 2010).

In terms of advertising language, Cao and Gao (2007) summarizes two general reasons for the emergence of vagueness. The copywriters apply vague language when (a) accurate descriptions or statements are impossible due to the nature of the products or services; and (b) special needs are to be met, such as decency or imaginativeness.

As for the theoretical framework of this paper, the pragmatic theory, Cooperative Principle and Conversational Implicature will be applied in the analysis of vagueness in commercial advertising. H. Paul Grice (1975) proposes the theory of Conversational Implicature, which describes how people interact with one another in conversation by working out the implied meaning behind the literal utterances. The concept "implicature" is advanced by him to indicate the inferred meaning. He makes a clear distinction between what is said and what is suggested, implied, meant, etc. He suggests there is some principle to govern the production and appreciation of the utterances in conversation, that is, the Cooperative Principle.

\section{Cooperative Principle}

Grice (1975) maintains that speakers generally intend to be cooperative in conversation. "Our talk exchanges do not normally consist of a succession of disconnected remarks, and would not be rational if they did. They are characteristically, to some degree at least, cooperative efforts". He states that "make your conversational contribution such as is required, at the stage at which it occurs, by the accepted purpose or direction of the talk exchange in which you are engaged" (ibid.). This is known as the Cooperative Principle. To illustrate this principle further, Grice puts forward four categories of maxims and the relevant sub-maxims:

\section{The Maxim of Quantity}

1. Make your contribution as informative as is required (for the current purposes of the exchange).

2. Do not make your contribution more informative than is required.

The Maxim of Quality

Try to make your contribution one that is true.

1. Do not say what you believe to be false.

2. Do not say that for which you lack adequate evidence.

The Maxim of Relation 
Be relevant.

The Maxim of Manner

Be perspicuous.

1. Avoid obscurity of expression.

2. Avoid ambiguity.

3. Be brief (avoid unnecessary prolixity).

4. Be orderly.

The maxims specify what speakers should do to converse successfully and efficiently. However, it should be pointed out that the maxims are not invariably followed. There are occasions on which one maxim is infringed for the purpose of conforming to another maxim, or flouted so as to get in a conversational implicature. In the latter situation, inferences about the speaker's true meaning are made by penetrating through the literal meaning of the utterances in order to adhere to the assumption of cooperation. Just as Grice puts it, some maxim is violated at the level of what is said, but that maxim, or at least the overall Cooperative Principle, is observed at the level of what is implicated.

\section{Positive Functions of Vague Language}

\subsection{Improving the Flexibility of Communication}

Vague language is conducive to the moderation of the atmosphere or tone. In advertising, copywriters tend to employ vague language when the products or services involve the topics that are generally considered unpleasant or offensive. By moderating the atmosphere or tone of communication, the copywriters are able to communicate in a flexible way.

\subsubsection{Improving the Flexibility of Communication by Displaying Politeness}

"Vague language softens expressions so that they do not appear too direct or unduly authoritative or assertive" (Carter \& McCarthy, 2006, cited in Cutting, 2007). In advertising, copywriters can apply vague language to display a euphemistic or polite atmosphere.

E.g. 1 Some ideas take time to develop.

Call the Webb Law Firm to protect your powerful ideas.

(Pittsburgh Post-Gazette, USA, 9 Jan 2012)

This is an advertisement for a law firm. After reading the first sentence, the readers may regard it as a kind of common knowledge because it is a fact in daily life. Then after finishing reading the second sentence, they may feel confused as they may not figure out why taking time to develop some ideas has something to do with calling the law firm. To offer a plausible reason to urge the readers to call the law firm, the copywriter might have stated the merits of the law firm to promote the firm. Therefore, the first and second sentences do not foster a direct causality and the advertisement flouts the maxim of relation.

To successfully sell the products or services, the job of the copywriters is to make sure the advertisements they present are effective in communication. By the same token, there is no reason to suppose that the copywriter in this advertisement does not observe the Cooperative Principle. In this sense, the readers presume the copywriter is cooperative and will endeavor to work out what is intended by the copywriter. They may interpret the advertisement as "some ideas take time to develop, and ideas related to laws are among them as they involve very professional knowledge. Call the Webb law firm and we will offer you professional service to protect your powerful ideas".

As is known, legal disputes are extremely unwelcome as they are time-consuming and costly. To openly demonstrate the merits of the law firm can be regarded offensive or unpleasant. On such an occasion where the euphemistic atmosphere is especially in need, the copywriter resorts to vagueness. By stating a common knowledge as a lead-in, the readers may first feel familiar and they may wonder why the ad puts it here. In this way, the readers' interest can be evoked. When the suggestion of calling the firm appears later, the readers tend to figure out the relation of the two sentences and work out the implicature. Hence, the copywriter's seeming flouting of the maxim of relation is caused for the purpose of conveying the message in a polite way. Vague language here is helpful to rendering the communication flexible.

Another similar example is:

E.g. 2 Plan your family night... 
Pick up the Saturday edition of Kokomo Tribune.

(Kokomo Tribune, USA, 5 Jan 2012)

This example also flouts the maxim of relation as the direct relation of "plan family night" and "pick up the newspaper" is not clearly revealed on the surface. The readers may work out the implicature in this way: while planning what your family is going to do on the weekend night, you may consider reading the Saturday edition of Kokomo Tribune. As weekends are conceived as valuable and private time, the copywriter applies vagueness so as to avoid directness which may risk being regarded as intruding the advertiser' s intention into the readers' freedom to decide how to spend the family night at weekend.

\subsubsection{Improving the Flexibility of Communication by Displaying Naturalness}

Vague language is useful in attaching naturalness to the tone of advertising. McCarthy (1998) holds that vague language makes "an important contribution to naturalness and the informal, convergent tenor of everyday talk" (Cited in Cutting, 2007). In the case that copywriters desire to render the advertisements more approachable, vague language may come into play.

E.g. 3 "I'll be on time, answer all your questions, and respect your home. I guarantee it."
Scott Goshern
Owner

(Kokomo Tribune, USA, 5 Jan 2012)

This is an advertisement for a business offering domestic services, including the maintenance of heating, cooling, plumbing, etc. Apparently, E.g. 3 is a promise made by the owner of the business. However, what may cause doubts is how it is possible for the owner to answer all the questions as he is supposed to be busy and the questions may differ from type to type. What " $\mathrm{I}$ " here refers to is vague and the ad is not as informative as is required. The example flouts the maxim of quality and quantity. What's more, the pronoun "it" is rather vague in the advertisement. Does it refer to the actions mentioned in the previous sentence, "be on time, answer all your questions, and respect your home", or something else?

As has been discussed before, the readers tend to figure out the implicature. The business concerned provides door-to-door maintenance service. Considering this, the readers can realize the personal pronoun "I" not only refers to the boss, but indicates the staff providing the maintenance work. What the owner says becomes the symbol of the promise made by the staff.

To directly inquire readers about their needs for domestic maintenance appears unwelcome as people normally expect their household facilities to function smoothly. Based on the nature of the business, the advertisement adopts the form of a daily dialogue to narrow the distance between the readers and the business. Making the promise in a conversational way is more natural and believable as the readers feel like hearing it face to face. "Answer all your questions" may appear excessively absolute, but as it is put in a daily conversation which is relatively casual rather than a formal writing which is thought to require more caution, it can be deemed appropriate and sincere.

As regards the pronoun "it", the vagueness it carries contributes to the informal, convergent tenor of the conversation. What "it" represents is supposed to be mutually known by both the copywriter and the readers. "It" may refer to the previous actions or a good quality in general, which is a shared knowledge as it is one of the most fundamental assurances a business is thought to offer to customers.

In sum, the vagueness in E.g. 3 adds naturalness and informality to the tone, by virtue of which the business can establish or strengthen its close- to- the -public image.

E.g. 4 Are you a fly-catcher, a super hero, or a starfish? We want to know!

(Kent Messenger Maidstone, UK, 6 Jan 2012)

E.g. 4 is an advertisement for beds. It flouts the maxim of relation as "fly-catcher", "super hero" and "starfish" are quite different things from beds and it is not plain why the advertiser wants to know whether the readers are such things. Based on the Cooperative Principle, the readers may interpret E.g. 4 like this: are you a fly-catcher, hero, or starfish? We (our beds) want to know, so let us (our beds) accompany you while you are experiencing magical things in your dreams. By exploiting vagueness, the copywriters try to promote beds in a natural and intimate way.

\subsection{Enhancing the Persuasiveness of Communication}

Schaff (1979) insists that our communication needs vague language. In his opinion, without all sorts of vague language, our means of communication would become so pale and their role in communication and expression 
limited to such a degree that our interchange is paralyzed (Cited in Xu, 2011). To promote the persuasiveness of communication, commercial advertising is in need of vague language.

\subsubsection{Enhancing the Expressiveness of Language}

Vague language diversifies our means of communication. It can "create more space for artistic description" (Zhang, 2012). In advertisements, vague language can play a positive role in enhancing the expressiveness of language through various ways.

E.g. 5 Our flowers last longer.

(The Advertiser State Edition, Australia, 9 Jan 2012)

This is an advertisement for a flower shop. It flouts the maxim of quality as the copywriter states something for which he lacks adequate evidence.

To understand what is intended by the copywriter, the readers may interpret the advertisement as: "Our flower shop is more careful and experienced in looking after the flowers, so our flowers have a better quality and last longer." Alternatively, associating the ad with the common purpose of buying flowers, they may understand it in this way: "Sending the flowers to the one I love or care, our love, as is symbolized by the flowers, will last longer." The readers may interpret the ad in either way to work out the implicature. In the latter case, flowers are compared to love. Hence, "Our flowers" here both refers to the concrete object, flowers sold by the shop, and the abstract feeling, love. In this way, E.g. 5 also flouts the maxim of manner that specifies the avoidance of ambiguity. The vague language here spurs persuasiveness in an artistic way.

E.g. 6 Give your life a lift.

(The Daily Telegraph, UK, 4 Jan 2012)

E.g. 6 is an advertisement for stairlifts, which are used for carrying a person who has difficulty in going up or down the stairs. The word "lift" not only refers to a machine that carries people, but also means "a feeling of being happier or more confident than before". Considering that people for whom the stairlifts are made have difficulty in action, "lift" in E.g. 6 probably involves these two meanings and the sentence flouts the maxim of manner that requires the avoidance of ambiguity. The vagueness makes the whole sentence more expressive.

\subsubsection{Enriching the Message of Ideas}

Kant holds that "vague ideas are more expressive than explicit ones... Beauty is inexpressible, and we cannot convey what we conceive in mind all the time" (Cited in Cao \& Gao, 2007). Vague language, by conveying ideas in an indefinite way, helps to maintain the full possibility of message that is encoded in the ideas, and gives readers more freedom to decode the possible message. "For the sake of economic principle, language needs to be highly generalized and vague to convey message to the greatest extent with a small quantity of signs" (Ye, 2012). Hence, vague language is capable of enriching the message with a limited range of signs.

\section{E.g. 7 When offroad riding is $6 \mathrm{~m}$ airborn}

(The Advertiser State Edition, Australia, 10 Jan 2012)

This is an advertisement for a motocross scene. The sentence is incomplete in structure. The syntactical vagueness causes the loss of part of the message. E.g. 7 flouts the maxim of quantity.

Motocross is an activity about extreme speed and excellent racing skills over rough ground. "One way to nourish desire is to help prospects visualize themselves enjoying the product or service" (Bovee \& Arens, 1992). As the advertisement makes a special reference to " $6 \mathrm{~m}$ airborne" and omits the following main clause, the readers are directed to imagine how they will be if they are at that height. Given the context, the readers are free to have their own versions of the main clause to dig out the implicature. As motocross is about pursuit of extreme feelings, which are emotional things and thus hard to be described by words, the vague language here, by triggering the imagination of the readers, conveys the wonderful feelings through a medium, that is, the readers' minds. Since these feelings are obtained via the images formed by the readers themselves, they may feel more convinced. Therefore, the vague language makes the advertisement more persuasive by enriching the message conveyed.

Other similar examples include:

E.g 8 If you haven't looked at Ford lately...

Look again.

(Qi, 2003) 
E.g. 9 Because every day is a family reunion

(Qi, 2003)

\subsection{Ensuring the Accuracy of Information}

\subsubsection{Vague Language and the Accuracy of Information}

In business, corporations generally endeavor to establish an honest and reliable public image for the sake of long-term benefits. Thus, in advertising, a channel of public communication, corporations also care about their images. The delivery of accurate and faithful information in advertising is a help to the corporations' reliable images.

Language is an essential carrier of information. In advertising, copywriters sometimes resort to vague language to ensure the accuracy of information. It may seem hard to understand the contribution of vague language to accurate information since "vagueness" and "accuracy" may be labeled as antonymous words. Nonetheless, vagueness is not definitely contradictory to accuracy. The word "accurate" means being correct and true in every detail, and "accuracy is a matter related to truth and falseness, which does not have to be shown through very precise manners, like quantity or other forms"( $\mathrm{Hu}, 2011)$. Copywriters may apply vague language to guarantee the accuracy of information under the circumstances where specific information is inadequate or special communicative purposes are desirable.

\subsubsection{Analysis of Vague Language's Role in Ensuring the Accuracy of Information}

Koester points out that vague language has transactional uses, which can be reflected in that vague language supplies the appropriate amount of information, obtains information or communicates effectively when specific information is lacking (Cited in Cutting, 2007).

E.g. 10 A new culture to discover,

a place that will take our breath away,

somewhere we can get lost,

then find ourselves again,

and won't cost the earth.

(The Independent, UK, 7 Jan2012)

This is an advertisement for a travelling agent. The expressions "a new culture", "a place", and "somewhere" are vague in meaning as their referents are unclear, and the expression "cost the earth" is also vague as the specific amount of money is not given. For readers who have intention to go travelling, the routes (the products) and the relevant prices are the basic information. However, the readers are not informed of these, so the example flouts the maxim of quantity.

For the travelling agent, it is no doubt that the agent itself knows the routes designed. Nonetheless, it cannot tell exactly which destination every prospect would like to go before his inquiring about the routes, nor can it provide beforehand the exact cost he may consume. Given the circumstances, the copywriter makes a general reference by saying "a new culture", "a place", "somewhere" to stand for the readers' ideal destinations, and uses "won't cost the earth" to indicate the reasonableness of the costs that are to be determined. Lacking the specific information of the readers' choices, the copywriter takes advantage of vague language to ensure the accuracy of information.

Vagueness stemming from the use of pronouns is also common in advertising to ensure the accuracy of information, such as

E.g. 11 ...After losing themselves in the sites and sounds, they can still find the way home. This holiday, give a Garmin.

(USA TODAY US Edition, USA, 28 Dec 2011)

E.g. 12 For one moment the world is spinning around her

(Qi, 2003)

Vague language can also be applied to protect the advertiser from undertaking too heavy responsibility, because the indeterminacy attached can lessen a certain degree of faithfulness of the advertisement, and thus lessens the degree of the advertiser's responsibility to maintain $100 \%$ faithfulness. Speakers may "employ vague language as a safeguard to guarantee that they are not wrong at any time" (Zhu, 2009). This is the same to advertisers. 


\section{E.g. 13 Don't miss out with up to $65 \%$ off}

(Kent Messenger Maidstone, UK, 6 Jan 2012)

"65\%", an eye-catching number, seems to specify the amount of the discount, but with the modifier "up to", meaning "as far as a particular number, level, etc.", the specific number loses its absolute specificity. The equivalent of the example can be: don't miss out with as much as but no more than $65 \%$ off. The specific number with the modifier "up to" is actually turned into a numerical range. Thus, the example is vague in meaning. Both " $10 \%$ " and " $65 \%$ " fall into the range of "up to $65 \%$ ", but the two figures differ greatly. Meanwhile, it is possible that merely a small part of the products reach the discount as high as $65 \%$ off. Thus, the readers cannot know clearly the proportion of the products at the discount as high as $65 \%$ off. E.g. 13 flouts the maxim of quantity.

Dyer (1995) asserts that the primary aim of advertising language is to attract our attention and dispose us favorably towards the product or service. The copywriter of E.g. 13 intends to enhance the attractiveness of the advertisement by showing the enticing figure of $65 \%$. Also, by using "up to", the copywriter demonstrates not all of the products reach this discount level. On the one hand, he works on the charm of the language to attract the readers' attention; on the other hand, he ensures the accuracy of the information by properly applying vague language. We can see that the vague language helps the copywriter to keep a good balance of exaggerated attractiveness and faithfulness.

Other similar examples are:

E.g. $140 \%$ purchase financing for up to 72 months

(Advertiser (Grand Falls), Canada, 19 Mar 2012)

E.g. 15 You're invited to save $30 \%$ or more

(The Advertiser State Edition, Australia, 29 Mar 2012)

\section{Negative Role of Vague Language}

Vague language in commercial advertising can play a positive role, as is analyzed above. However, it can have negative effects as well if not appropriately applied. Although the purpose of commercial advertisements is to promote goods or services with the expectation of making a profit, some advertisements may risk promoting goods or services in a bad way. "The vagueness device in advertising language brings about negative consequences when abusively or improperly used, under which circumstances the customers are directed to false understanding or misled" (Xie \& Liu, 1997).

E.g. 16 Only $\$ 1,996$, unbeatable value

(Herald Sun, Australia, 16 Dec 2011)

"Unbeatable", meaning "impossible to defeat or improve", shows that the value has reached the level that is impossible to be surpassed. It is plain that E.g. 16 flouts the maxim of quality as the copywriter states something for which he lacks adequate evidence.

What's intended by the copywriter is that he wants to emphasize the tantalizing price, which he thinks is more reasonable than those of other competitors. Some readers may be attracted and purchase the advertised product. However, if they find out later the business itself or other competitors offer the same thing at a lower price, they may probably consider the ad misleading. Following this, the credibility of the business may be undermined.

Another typical example in daily life is:

E.g. 17 Buy one, and get one for free.

It is sometimes the case that the advertised thing is bought, and what is given for free is a different thing. This example flouts the maxim of quality, as the copywriter states something he believes to be false, or flouts the maxim of manner, as the pronoun "one" causes ambiguity. The readers under such conditions are directed to have false understanding by the vague language.

\section{Conclusion}

As is shown above, the study first demonstrates the connection between commercial advertising and language, and points out that vagueness is typical in advertising language which is loaded language. Then the definition of vague language is explored, which has not received a unanimous agreement. In this paper, vague language is considered as the language property of indeterminacy. Afterwards, the causes of vague language are shown.

This paper revolves around the functions of vague language in commercial advertising and the motives of 
copywriters to apply that. The paper finds that copywriters resort to vague language under such circumstances: (a) the advertisements risk being deemed as offensive or unnatural; (b) the copywriters seek for greater persuasiveness; and (c) the copywriters are short of specific information related to consumers or a balance of attractiveness and faithfulness is pursued. Based on the pragmatic theory, Cooperative Principle and Conversational Implicature, both the positive and negative roles vague language plays are anatomized. The paper finds that when properly applied, vague language in advertisements is conducive to the flexibility of communication by displaying politeness and naturalness, to the persuasiveness of communication by enhancing the expressiveness of language and enriching the message of ideas, as well as to the accuracy of information. However, if inappropriately exploited, vague language may mislead or cause the readers to have false interpretations of the advertisements.

\section{References}

Advertiser (Grand Falls), 19 Mar 2012, p. A9.

Bovee C., \& Arens W. (1992). Contemporary Advertising (4th ed.). U.S.: Von Hoffmann Press, Inc.

Cao Wei, \& Gao Jun. (2007). Advertising Linguistics: a Course Book. Guangzhou: Jinan University Press.

Channell J. (2000). Vague Language. Shanghai: Shanghai Foreign Language Education Press.

Cutting J. (Eds.). (2007). Vague Language Explored. New York: Palgrave Macmillan. http://dx.doi.org/10.1057/9780230627420

Dyer G. (1995). Advertising as Communication (7th ed.). London: Routledge.

Grice, Paul. (1975). Logic and conversation. In P. Cole, \& J. Morgan (Ed.), Syntax and Semantics, 3: Speech Acts. New York: Academic Press.

Herald Sun, 16 Dec 2011, p. A11.

He Ziran. (2000). A Further Study on Pragmatic Vagueness. Journal of Foreign Languages, 125(1), 7.

Hu Weiwei. (2011). A Study on the Reasonableness of Vague Language in News Reports. News Research, 12, 106.

Kent Messenger Maidstone, 6 Jan 2012, pp. A17, A24.

Koester A. About Twelve Thousand or So: Vagueness in North American and UK Offices. In J. Cutting (Ed.), Vague Language Explored (2007, p. 44). New York: Palgrave Macmillan.

Kokomo Tribune, 5 Jan 2012, p. A2.

Pittsburgh Post-Gazette, 9 Jan 2012, p. A8.

Qiao Zehui. (2010). Study on the Use of Fuzzy Language in Business English Context. M.A. Beijing Forestry University.

Qi Yunfang (Eds.). (2003). Advertisement and English in Advertising. Zhejiang: Zhejiang University Press.

The Advertiser State Edition, 9 Jan 2012, p. A3.

The Advertiser State Edition, 10 Jan 2012, p. A23.

The Advertiser State Edition, 29 Mar 2012, p. A10.

The Daily Telegraph, 4 Jan 2012, p. A31.

The Independent, 7 Jan2012, p. A30.

Trappes-Lomax H. Vague Language as a Means of Self-Protective Avoidance: Tension Management in Conference Talks. In J. Cutting (Ed.), Vague Language Explored (2007, p. 122). New York: Palgrave Macmillan.

USA TODAY US Edition, 28 Dec 2011, pp. A4, A5.

Xie Wenyi, \& Liu Yunteng (Eds.). (1997). Advertising English. Shanghai: Shanghai Jiao Tong University Press.

Xu Yiping. (2011). On Pragmatic Functions of Fuzziness in English Advertisements. Journal of Longyan University, 29(6), 68.

Ye Youzhen. (2012). Question on the Assumption of Fussiness of Meaning. Journal of Tianjin Foreign Studies University, 19(5), pp. 39, 43. 
Zhang Hui. (2012). Translation Studies and Fuzzy Language in Guo Moruo's Artistic Essays. Foreign Language and Literature (bimonthly), 28(3), 107.

Zhu Jie. (2009). A Tentative Study on Pragmatic Functions of Vague Language. Ph.D. Fudan University. 